\title{
A combined study of the pion's static properties and form factors
}

Version of April 1, 2022

\begin{abstract}
We study consistently the pion's static observables and the elastic and $\gamma^{*} \gamma \rightarrow \pi^{0}$ transition form factors within a light-front model. Consistency requires that all calculations are performed within a given model with the same and single adjusted length or mass-scale parameter of the associated pion bound-state wave function. Our results agree well with all extent data including recent Belle data on the $\gamma^{*} \gamma \rightarrow \pi^{0}$ form factor at large $q^{2}$, yet the BaBar data on this transition form factor resists a sensible comparison. We relax the initial constraint on the bound-state wave function and show the BaBar data can partially be accommodated. This, however, comes at the cost of a hard elastic form factor not in agreement with experiment. Moreover, the pion charge radius is about $40 \%$ smaller than its experimentally determined value. It is argued that a decreasing charge radius produces an ever harder form factor with a bound-state amplitude difficultly reconcilable with soft QCD. We also discuss why vector dominance type models for the photon-quark vertex, based on analyticity and crossing symmetry, are unlikely to reproduce the litigious transition form factor data.
\end{abstract}

Keywords Neutral Pion · Axial anomaly · Form factors · Light-Front Field Theory

\section{Introduction}

The transition $\gamma^{*} \gamma \rightarrow \pi^{0}$ has attracted considerable attention with the experimental findings of the BaBar Collaboration [1]. Indeed, while this new data set appears to agree with earlier experiments on a domain of squared-momentum transfer below $Q^{2}=-q^{2} \lesssim 10 \mathrm{GeV}^{2}[2 ; 3]$, the data points at larger $Q^{2}$ values remarkably exceed the prediction of perturbative QCD (pQCD) in the asymptotic limit $[4 ; 5]$. On the other hand, a most recent measurement by Belle [6] appears to corroborate the pQCD limit. The process $\gamma^{*} \gamma \rightarrow \pi^{0}$ is by itself of highly phenomenological and theoretical interest. If the entire domain of experimentally explored squared-momentum transfer is to be described within a unique theoretical framework, it must account for the mainly nonperturbative phenomenon of the Abelian anomaly and functional behavior of perturbative QCD. This is the challenge for any model employed in a self-consistent way.

Herein we present the results of a consistent treatment of the pion's static features as well as of the electromagnetic and $\gamma^{*} \gamma \rightarrow \pi^{0}$ transition form factors within one particular model, namely the

Bruno El-Bennich (E-mail: bruno.bennich@cruzeirodosul.edu.br)

Laboratorio de Física Teórica e Computacional, Universidade Cruzeiro do Sul, 01506-000, São Paulo, Brazil and Instituto de Física Teórica, Universidade Estadual Paulista, 01140-070, São Paulo, Brazil.

João Pacheco B. C. de Melo

Laboratorio de Física Teórica e Computacional, Universidade Cruzeiro do Sul, 01506-000, São Paulo, Brazil.

Tobias Frederico

Departamento de Física, Instituto Tecnológico de Aeronáutica, CTA, 12228-900, Saõ José dos Campos, Brazil. 
light-front model introduced in Ref. $[7 ; 8]$ and refined in later studies $[9 ; 10 ; 11 ; 12 ; 13 ; 14 ; 15]$. This work differs from several recent attempts $[16 ; 17 ; 18 ; 19 ; 20 ; 21 ; 22 ; 23 ; 24 ; 25 ; 26 ; 27]$ to reproduce the large- $Q^{2}$ BaBar data in spirit and in aim: we do not attempt to build a phenomenological model that yields a good fit to the BaBar data. Rather, within the framework of the present model, we insist on a consistent and simultaneous treatment of all extent data, not merely the $\gamma^{*} \gamma \rightarrow \pi^{0}$ transition form factor.

We first calculate the static properties, the pion decay constant and electric charge radius. The pion decay constant, $f_{\pi}$, serves to adjust the unique parameter that enters the bound-state wave function, $r_{\mathrm{nr}}$, and introduces a length (or mass) scale. This immediately fixes the root-mean-square (rms) charge radius of the pion whose known experimental value, $\left\langle r_{\pi}^{2}\right\rangle^{1 / 2}=0.672 \pm 0.008 \mathrm{fm}$ [28], can be compared with. Next, with this parametrized bound state function, we calculate the leading contribution to the electromagnetic form factor, $F_{\pi}\left(Q^{2}\right)$, and to the transition form factor, $F_{\gamma \pi}\left(Q^{2}\right)$, in the impulse approximation on the light cone. The main freedom in these calculations is, of course, the choice of bound-state wave function or in other words, the associated Bethe-Salpeter amplitude.

We devise a wave function which, in interplay with our light-front quark model, reproduces the experimental space-like tail of $F_{\pi}\left(Q^{2}\right)$ and thus the asymptotic limit of the product $Q^{2} F_{\pi}\left(Q^{2}\right) \rightarrow$ $16 \pi f_{\pi}^{2} \alpha_{s}\left(Q^{2}\right)$ for $Q^{2} \rightarrow \infty[4 ; 5]$. It should be stressed that the decay constant and charge radius are insensitive to the asymptotic form of the wave function. This is the reason for the success of quark models or related contact-interaction models in the calculation of the pion's static properties below a mass scale $M^{2}$ [29]. However, probing the pion with $Q^{2} \gtrsim M^{2}$ leads to marked deviations from experiment when the contact-interaction model is treated self-consistently with proper regularization $[30 ; 31 ; 32]$. In particular, due to the lack of a running mass function, models based on constituent quarks must necessarily introduce a phenomenological bound-state amplitude that mimics both soft QCD effects and the asymptotic behavior of the associated pion distribution function, $\phi_{\pi}(x)$. Therefore, this process also allows us to scrutinize and improve the description of the pion bound-state function in relativistic light-cone models.

To summarize, we first describe the light-front formulation of the $\gamma^{*} \gamma \rightarrow \pi^{0}$ and introduce the parametrization of the pion bound-state wave function in Sec. 2. The values we obtain from this parametrization for $F_{\gamma \pi}\left(Q^{2}\right)$ and $F_{\pi}\left(Q^{2}\right)$ as well as the pion charge radius and decay constant are discussed in Sec. 3. We resume in Sec. 4 where we relax the condition on the length scale parameter and observe the implication on the functional behavior of $F_{\gamma \pi}\left(Q^{2}\right)$, in particular whether the asymptotic prediction of perturbative QCD is still verified. We also check the values obtained in this case for $\left\langle r_{\pi}^{2}\right\rangle^{1 / 2}$ and $f_{\pi}$ and modifications occurring in $F_{\pi}\left(Q^{2}\right)$. In Sec. 5 we wrap up with a qualitative discussion of the $\gamma^{*} \gamma \rightarrow \pi^{0}$ form factor and its possible evolution upon the inclusion of vector resonances in the deep space-like region.

\section{Pion form factors on the light-front}

\subsection{Preliminaries}

The geometry employed, for example by the BaBar Collaboration, to produce the $\pi^{0}$ consists of an untagged almost real $\left(k_{2}^{2} \approx 0\right)$ photon scattered at small angle from the collision axis and a tagged electron emitting a highly off-shell photon with space-like momentum $k_{1}$. The two photons eventually create the neutral pion via the Abelian anomaly.

The relativistic approach to the wave function based on constituents quarks is possible due to the absence of pair-creation processes on the light front. This salient feature arises from the particular choice of the light-front coordinates [33; 34; 35], defined by $x^{+}=x^{0}+x^{3}$, in which the center-of-mass is readily separated [36]. In essence, the impulse approximation depicted in Fig. 1 involves a bound state and an internal loop momentum. The integration over the loop momentum is first performed in the convergent light-front energy, $k^{-}$, after which the pion's wave function emerges naturally within the three-dimensional remainder of the integral and depends on the + and $\perp$ component of the $\bar{q} q$ relative momentum $[7 ; 8 ; 9 ; 10 ; 37]$. We stress that in defining a model for this light-front wave function, the convergence of the $k^{-}$integration is crucial: it is the case for the good component of the current [33; 34] and also for the diagram that describes the weak decay constant. 


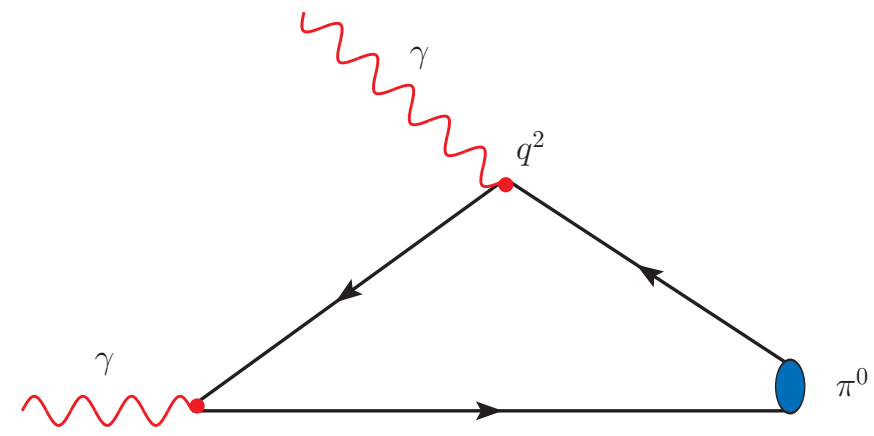

Fig. 1 The leading diagram corresponding to the impulse approximation of the process $\gamma^{*} \gamma \rightarrow \pi^{0}$. The lines with arrows denote constituent light quarks; the solid blobs represent bare photon-quark vertices and the filled oval depicts the $\bar{q} q$ bound-state wave function of the pion.

The aim of this paper is to study models of light-front wave functions in computations of the electromagnetic and transition form factors of the neutral pion with simultaneous verification of the related weak decay constant and charge radius. We make use of two distinct model wave function:

i) a Gaussian model [38; 39];

ii) a hydrogen-type wave function [40].

Both wave functions feature a length or mass scale parameter, $r_{\mathrm{nr}}$, whose value is of the order of $\Lambda_{\mathrm{QCD}}$. We fix this scale with a fit to the pion decay constant, all other observables follow from this parametrization without exception. We compare the results for the form factors obtained with these two wave functions in Sec. 3 .

\subsection{Formulation of light-front amplitudes and form factors}

In the following, we formulate the $\gamma^{*} \gamma \rightarrow \pi^{0}$ transition form factor in the light-front approach. The coupling of the pseudoscalar $\bar{q} q$ pair to the pion is expressed through the following effective Lagrangian $[7 ; 8]$,

$$
\mathcal{L}_{\pi q}^{\mathrm{int}}=-\imath \frac{M}{f_{\pi}} \pi \cdot \bar{q} \gamma^{5} \tau q
$$

where $M$ is the constituent-quark mass, $f_{\pi}=92.4 \mathrm{MeV}$ is the weak decay constant of the pion, $\boldsymbol{\pi}$ and $q$ are respectively the pion field and quark wave functions. The units are chosen such that $\hbar=c=1$. This coupling can be thought of as the leading term of the full pseudoscalar Bethe-Salpeter amplitude.

The amplitude for the electromagnetic process $\gamma^{*} \gamma \rightarrow \pi^{0}$ in the impulse approximation is diagrammatically represented in Fig. 1 and given by the tensor, $T^{\mu \nu}$, which contains two contributions due to bosonic symmetrization of the amplitude:

$$
T_{\mu \nu}\left(k_{1}, k_{2}\right)=t_{\mu \nu}\left(k_{1}, k_{2}\right)+t_{\mu \nu}\left(k_{2}, k_{1}\right) \text {. }
$$

The tensor $t_{\mu \nu}\left(k_{1}, k_{2}\right)$ is obtained after taking the traces in spinor and flavor space [41]:

$$
t_{\mu \nu}=\frac{4}{3} \frac{M^{2}}{f_{\pi}} e_{0}^{2} N_{c} \epsilon_{\mu \nu \alpha \beta} k_{1}^{\alpha} k_{2}^{\beta} I\left(k_{1}^{2}\right)
$$

where $I\left(k_{1}^{2}\right)$ is the scalar loop integral,

$$
I\left(k_{1}^{2}\right)=\int \frac{d^{4} k}{(2 \pi)^{4}} \frac{1}{\left(\left(k_{2}-k\right)^{2}-M^{2}+\imath \epsilon\right)} \frac{1}{\left(k^{2}-M^{2}+\imath \epsilon\right)\left(\left(k_{\pi}-k\right)^{2}-M^{2}+\imath \epsilon\right)} .
$$

Here, $N_{c}$ is the number of colors, $k_{\pi}=k_{1}+k_{2}$ is the $\pi^{0}$ momentum, $k_{1} \equiv q$ is the space-like momentum transfer, $k_{2}^{2}=0$ is the on-shell photon and $e_{0}$ and $M$ are the unit charge and constituent quark mass, respectively. The factor $1 / 3$ in Eq. (3) stems from the flavor trace. 
After transformation to light-front variables, $\mathbf{k}_{\perp}, k^{+}=k^{0}+k^{3}$ and $k^{-}=k^{0}-k^{3}$, we first integrate over $k^{-}$, the light-front energy. The reference frame is chosen such that $q^{+}=q^{-}=0$ and momentum transfer is transversal, $q_{\perp}$, which is always possible due to the space-like character of $q$. In this coordinate system, and after factorizing $k^{+}, k_{\pi}^{+}-k^{+}$and $k_{2}^{+}-k^{+}$in the denominator, the integral in Eq. (4) becomes

$$
\begin{aligned}
I\left(q^{2}\right) & =\frac{d^{4} k}{2(2 \pi)^{4}} \int d k^{-} d k^{+} d^{2} k_{\perp} \frac{1}{k^{+}\left(k_{\pi}^{+}-k^{+}\right)\left(k_{2}^{+}-k^{+}\right)\left(k^{-}-\frac{k_{\perp}^{2}+M^{2}-\imath \epsilon}{k^{+}}\right)} \\
& \times \frac{1}{\left(k_{2}^{-}-k^{-} \frac{\left(\mathbf{k}_{2}-\mathbf{k}\right)_{\perp}^{2}+M^{2}-\imath \epsilon}{\left(k_{2}^{+}-k^{+}\right)}\right)\left(k_{\pi}^{-}-k^{-} \frac{\left(\mathbf{k}_{\pi}-\mathbf{k}\right)_{\perp}^{2}+M^{2}-\imath \epsilon}{\left(k_{\pi}^{+}-k^{+}\right)}\right)}
\end{aligned}
$$

where $k_{\pi}^{+}=k_{2}^{+}$and $k_{\pi}^{-}=k_{2}^{-}$in this reference frame.

The integration in $k^{-}$, performed using Cauchy's theorem, is convergent. The position of the poles in the $k^{-}$complex plane depends on the value of $k^{+}$. Since $k_{\pi}^{+}=k_{2}^{+}$, this integral is non-vanishing in only one region of $k^{+}$. The set $k^{+}<0$ and $k^{+}>k_{\pi}^{+}$does not contribute to the integral because the three poles in there have imaginary parts with same signs. The only contribution comes from the region where the + component of the quark momentum cannot exceed the pion momentum: $0<k^{+}<k_{\pi}^{+}$. This corresponds to an on-shell quark. The result is

$$
I\left(q^{2}\right)=\frac{-\imath}{2(2 \pi)^{3}} \int \frac{d x d^{2} k_{\perp}}{x(1-x)^{2}} \frac{1}{\left(k_{2}^{-} k_{2}^{+}-\frac{k_{\perp}^{2}+M^{2}}{x}-\frac{\left(\mathbf{k}_{2}-\mathbf{k}\right)_{\perp}^{2}+M^{2}}{1-x}\right)\left(k_{\pi}^{-} k_{\pi}^{+}-\frac{k_{\perp}^{2}+M^{2}}{x}-\frac{\left(\mathbf{k}_{\pi}-\mathbf{k}\right)_{\perp}^{2}+M^{2}}{1-x}\right)},
$$

with the momentum fraction, $x=k^{+} / k_{\pi}^{+}$, and $0<x<1$.

Introducing in Eq. (6) the relative transverse $\bar{q} q$ momentum, as defined in Ref. [36],

$$
\mathbf{K}_{\perp}=(1-x) \mathbf{k}_{\perp}-x\left(\mathbf{k}_{\pi}-\mathbf{k}\right)_{\perp}
$$

we have

$$
I\left(q^{2}\right)=\frac{\imath}{2(2 \pi)^{3}} \int \frac{d x d^{2} K_{\perp}}{x(1-x)} \frac{1}{\left((\mathbf{K}-x \mathbf{q})_{\perp}^{2}+M^{2}\right)\left(m_{\pi}^{2}-M_{0}^{2}\right)},
$$

where $m_{\pi}$ is the pion mass. The free-mass operator for $\bar{q} q$ pair is written in terms of the momentum fraction, $x$, and the relative perpendicular momentum as:

$$
M_{0}^{2}\left(K_{\perp}^{2}, x\right)=\frac{K_{\perp}^{2}+M^{2}}{x(1-x)} .
$$

The matrix element of the neutral pion decay driven by the Abelian anomaly is given by one unique $C P T$-invariant Lorentz structure. When one of the photons is off-shell the same matrix element describes the transition amplitude $\gamma^{*} \gamma \rightarrow \pi^{0}$,

$$
\left\langle\pi^{0}\left(k_{\pi}\right)\left|J_{\mu}^{\pi^{0}}\right| \gamma\left(k_{2}\right)\right\rangle=e^{2} \epsilon_{\mu \nu \alpha \beta} \epsilon_{\gamma}^{\nu} q^{\alpha} k_{\pi}^{\beta} F_{\gamma \pi^{0}}\left(q^{2}\right),
$$

where $\epsilon_{\gamma}^{\nu}$ is the polarization of the real photon. Using Eqs. (2), (3) and (8), the transition form factor is given in our model by,

$$
F_{\gamma \pi^{0}}\left(q^{2}\right)=\frac{N_{c}}{6 \pi^{3}} \frac{M^{2}}{f_{\pi}} \int \frac{d x d^{2} K_{\perp}}{x(1-x)} \frac{1}{\left((\mathbf{K}-x \mathbf{q})_{\perp}^{2}+M^{2}\right)\left(m_{\pi}^{2}-M_{0}^{2}\right)} .
$$

In the soft pion limit, following Ref. [41], the form factor becomes

$$
F_{\gamma \pi^{0}}(0)=\frac{1}{4 \pi^{2} f_{\pi}}
$$

and the neutral pion decay width is given by [41],

$$
\Gamma_{\pi}^{0}=\frac{\alpha^{2} m_{\pi}^{3} \pi}{4} F_{\gamma \pi^{0}}^{2}(0),
$$


where $\alpha$ is the fine structure constant. A charge radius, $\sqrt{\left\langle r_{\pi^{0}}^{2}\right\rangle}$, can be defined via,

$$
r_{\pi^{0}}^{2}=\left.6 \frac{d F_{\gamma \pi^{0}}\left(q^{2}\right)}{d q^{2}}\right|_{q^{2}=0}
$$

which in the soft-pion limit behaves as [42],

$$
\sqrt{\left\langle r_{\pi^{0}}^{2}\right\rangle}=\frac{1}{\sqrt{2} M}
$$

To complete this section on form factors, we recall the long-standing result on the light front for the elastic pion form factor [7]. Its expression is likewise obtained following the steps of Eqs. (4) to (8) with the definition of the elastic form factor $\left(q=p^{\prime}-p\right)$ :

$$
T_{\mu}\left(p, p^{\prime}\right)=\left\langle\pi^{+}\left(p^{\prime}\right)\left|J_{\mu}^{\pi^{+}}\right| \pi^{+}(p)\right\rangle=e F_{\pi}\left(q^{2}\right)\left(p+p^{\prime}\right)_{\mu} .
$$

The elastic form factor is thus given by

$$
F_{\pi}\left(q^{2}\right)=\frac{2 N_{c}}{(2 \pi)^{3}} \frac{M^{2}}{f_{\pi}^{2}} \int \frac{d x d^{2} K_{\perp}}{x(1-x)} \frac{M_{0}^{2}\left[1+\frac{(1-x) \mathbf{q}_{\perp} \cdot \mathbf{K}_{\perp}}{\mathbf{K}_{\perp}^{2}+M^{2}}\right]}{\left(m_{\pi}^{2}-M_{0}^{\prime 2}\right)\left(m_{\pi}^{2}-M_{0}^{2}\right)}
$$

where the free mass operators, $M_{0}^{2}\left(K_{\perp}^{2}\right)$ and $M_{0}^{\prime 2}\left(K_{\perp}^{\prime 2}\right)$, were defined in Eq. (9) in terms of $x$ and the relative perpendicular momenta, $\mathbf{K}_{\perp}=(1-x)(\mathbf{p}-\mathbf{k})_{\perp}-x \mathbf{k}_{\perp}$ and $\mathbf{K}_{\perp}^{\prime}=\mathbf{K}_{\perp}+(1-x) \mathbf{q}_{\perp}$. The expression for the rms charge radius, in the soft-pion limit,

$$
\sqrt{\left\langle r_{\pi}^{2}\right\rangle}=\frac{\sqrt{3}}{2 \pi} \frac{1}{f_{\pi}}
$$

is know as the Tarrach relation [43].

\subsection{Wave function models}

We can identify an asymptotic pion wave function in Eqs. (11) and (17) and proceed to model one which reproduces soft QCD at low momentum transfer and hard perturbative effects for large $q^{2}$. We follow Ref. [7] where the following replacement is in order,

$$
\frac{1}{-m_{\pi}^{2}+M_{0}^{2}} \longrightarrow \frac{\pi^{\frac{3}{2}} f_{\pi}}{M \sqrt{M_{0} N_{c}}} \Phi_{\pi}\left(K^{2}\right)
$$

and the wave-function $\Phi\left(K^{2}\right)$ is normalized to one:

$$
\int d^{3} K \Phi_{\pi}^{2}\left(K^{2}\right)=1
$$

This is the normalization condition within the framework of Hamiltonian light-front dynamics [37; 44]. The functional dependence of $K^{2}$ on $\mathbf{K}_{\perp}$ and $x$ originates in the free mass operator, $M_{0}^{2}$,

$$
K^{2}\left(\mathbf{K}_{\perp} ; x\right)=\frac{M_{0}^{2}}{4}-M^{2}
$$

From Eq. (11) and Eqs. (19) to (21), we obtain the integral expression for the neutral pion transition form factor on the light front,

$$
F_{\gamma \pi^{0}}\left(q^{2}\right)=\frac{\sqrt{N_{c}} M}{6 \pi^{\frac{3}{2}}} \int \frac{d x d^{2} K_{\perp}}{x(1-x) \sqrt{M_{0}}} \frac{\Phi_{\pi}\left(K^{2}\right)}{(\mathbf{K}-x \mathbf{q})_{\perp}^{2}+M^{2}},
$$

In the limit $Q^{2}=-q^{2} \rightarrow \infty$, pQCD predicts the form factor decreases asymptotically as $\sim q^{-2}$ with a limiting value, $Q^{2} F_{\gamma \pi^{0}}\left(Q^{2}\right)=2 f_{\pi}[4 ; 5]$. 
Table 1 The model's length scale parameter, $r_{\mathrm{nr}}$ [Eqs. (26) and (27)], as a function of the constituent quark masses and for $f_{\pi}=92.4 \mathrm{MeV}$ given by Eq. (23). The corresponding charge radii are listed next to $r_{\mathrm{nr}}$ for both the neutral and charged pion.

\begin{tabular}{|c|c|c|c|c|}
\hline Model & $m_{u, d}[\mathrm{GeV}]$ & $r_{\mathrm{nr}}[\mathrm{fm}]$ & $\left\langle r_{\pi}^{2}\right\rangle^{1 / 2}[\mathrm{fm}]$ & $\left\langle r_{\pi^{0}}^{2}\right\rangle^{1 / 2}[\mathrm{fm}]$ \\
\hline Gaussian & 0.220 & 0.345 & 0.637 & 0.683 \\
& 0.330 & 0.472 & 0.655 & 0.552 \\
\hline Hydrogen & 0.220 & 0.593 & 0.795 & 0.782 \\
& 0.330 & 0.708 & 0.807 & 0.582 \\
\hline Experiment [28] & & & $0.672 \pm 0.008$ & \\
\hline
\end{tabular}

Table 2 The models' length scale parameter, $r_{\mathrm{nr}}$ [Eqs. (26) and (27)], and corresponding pion charge radii for increasing values of the pion decay constant $f_{\pi}$.

\begin{tabular}{|c|c|c|c|c|c|}
\hline Model & $f_{\pi}[\mathrm{MeV}]$ & $m_{u, d}[\mathrm{GeV}]$ & $r_{\mathrm{nr}}[\mathrm{fm}]$ & $\left\langle r_{\pi}\right\rangle^{1 / 2}[\mathrm{fm}]$ & $\left\langle r_{\pi^{0}}\right\rangle^{1 / 2}[\mathrm{fm}]$ \\
\hline Gaussian & 92.4 & 0.220 & 0.345 & 0.637 & 0.683 \\
& 97.0 & 0.220 & 0.303 & 0.589 & 0.657 \\
& 110.0 & 0.220 & 0.172 & 0.406 & 0.564 \\
\hline Hydrogen & 92.4 & 0.220 & 0.593 & 0.795 & 0.782 \\
& 97.0 & 0.220 & 0.543 & 0.750 & 0.767 \\
& 110.0 & 0.220 & 0.410 & 0.626 & 0.720 \\
\hline Experiment [28] & & & & $0.672 \pm 0.008$ & \\
\hline
\end{tabular}

For the purpose of completeness, we also quote the result for the neutral pion decay, $k_{1}^{2}=k_{2}^{2}=0$. The amplitude for this process can be formulated using the same reasoning that led to Eq. (22),

$$
F_{\pi^{0} \rightarrow \gamma \gamma}=\frac{2}{3} \frac{\sqrt{N_{c}} M}{\pi^{3 / 2}} \int \frac{d x d^{2} K_{\perp}}{x(1-x) \sqrt{M_{0}}} \frac{1}{\left(\left(\mathbf{K}-2 x k_{\gamma}\right)_{\perp}^{2}+M^{2}\right)} \Phi_{\pi}\left(K^{2}\right),
$$

where $\left|k_{\gamma}\right|=m_{\pi} / 2$ and $x \leq 1 / 2$. In the soft-pion limit and for a constant pion vertex, $\Phi_{\pi} \sim\left(-m_{\pi}^{2}+\right.$ $\left.M_{0}^{2}\right)^{-1}$,

$$
F_{\pi^{0} \rightarrow \gamma \gamma}=F_{\gamma \pi^{0}}\left(Q^{2}=-q_{\perp}^{2}=0\right)
$$

In practice, the calculation of the neutral pion width using either $F_{\gamma \pi^{0}}(0)$ in Eq. (22) or $F_{\pi^{0} \rightarrow \gamma \gamma}$ in Eq. (23) yield the same numerical result within the light-front approach. This follows from the observation that the pion mass is much smaller than the inverse of the characteristic length or mass scale of the wave function.

As mentioned earlier, both form factors are calculated with two different $\bar{q} q$ bound-state wave functions: the Gaussian and the hydrogen-atom model. Both models depend on two parameters, the constituent quark mass, $M$ and the length (or mass) scale, $r_{\mathrm{nr}}$, of the wave function. In order to test the model's mass dependence, all calculations are done with two constituent-quark masses for which we choose $M=m_{u, d}=220 \mathrm{MeV}$ and $330 \mathrm{MeV}$. This length (or mass) scale is set by fitting the pion decay constant, $f_{\pi}=92.4 \mathrm{MeV}[9]$,

$$
f_{\pi}=\frac{\sqrt{N_{c}} M}{4 \pi^{3 / 2}} \int \frac{d x d^{2} k_{\perp}}{x(1-x) \sqrt{M_{0}}} \Phi_{\pi}\left(k^{2}\right)
$$

where the two models for the pion wave function are explicitly given by,

$$
\begin{aligned}
& \Phi_{\pi}\left(K^{2}\left(\mathbf{K}_{\perp} ; x\right)\right)=\mathcal{C}_{\pi} \exp \left[-\frac{4}{3} r_{\mathrm{nr}}^{2} K^{2}\right], \\
& \Phi_{\pi}\left(K^{2}\left(\mathbf{K}_{\perp} ; x\right)\right)=\mathcal{C}_{\pi} \frac{1}{\left(r_{\mathrm{nr}}^{2}+K^{2}\right)^{2}},
\end{aligned}
$$




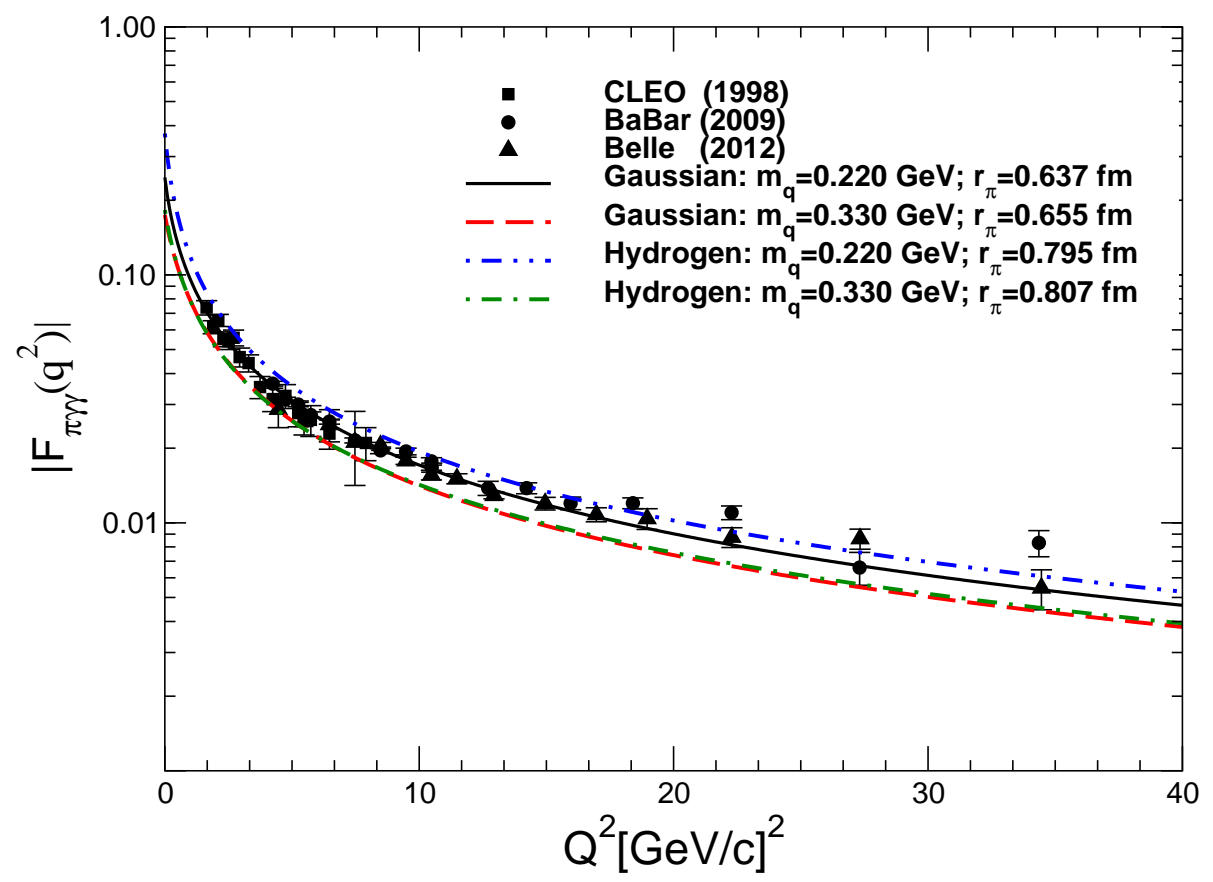

Fig. 2 The space-like $\gamma^{*} \gamma \rightarrow \pi^{0}$ transition form factor, $F_{\gamma \pi}\left(Q^{2}=-q^{2}\right)$, for two different constituent quark masses $m_{q}=m_{u, d}=M$ and $f_{\pi}=92.4 \mathrm{MeV}$; see Eqs. (26) and (27) for the two model wave functions and Table 1 for the corresponding length scale parameter, $r_{\mathrm{nr}}$, and charge radii. Note that the ordinate is scaled logarithmically. Data are from Refs. $[1 ; 3 ; 6]$.

where $\mathcal{C}_{\pi}$ is the overall normalization. Once $r_{\mathrm{nr}}$ is fixed, the charge radius (14), the transition form factor (22), and the elastic form factor (17) are all calculated with the same given pion wave function on the light cone. In Section 3, we explore the model sensitivity to the constituent quark mass with regard to the pion's static observables and elastic and transition form factors. In Section 4 we study the impact of modifying the pion's length scale $r_{n r}$ on the functional behavior of $F_{\gamma \pi^{0}}\left(Q^{2}\right)$ and the consequences for $F_{\pi}\left(Q^{2}\right)$ and the rms charge radius.

\section{Numerical results and variation of the constituent quark mass}

Numerical results for the transition form factor, $F_{\gamma \pi^{0}}\left(Q^{2}\right)$, and its weighted description, $Q^{2} F_{\gamma \pi^{0}}\left(Q^{2}\right)$, are presented in Figs. 2 and 3 for the choice $M=m_{u, d}=220 \mathrm{MeV}$ and $330 \mathrm{MeV}$ of constituent quark masses. The length scale, $r_{\mathrm{nr}}$, is adjusted in both cases so as to reproduce the pion decay constant in Eq. (25). As seen from Table 1, the calculated rms charge radius is in agreement (but too small) with the experimental value for the Gaussian case but about $20 \%$ larger when using the hydrogen model. In the asymptotic limit, pQCD predicts an upper bound $\lim _{Q^{2} \rightarrow \infty} Q^{2} F_{\gamma \pi^{0}}\left(Q^{2}\right)=2 f_{\pi}=0.185 \mathrm{GeV}$; Fig. 3 gives the impression that in all but one case the curves are not bounded by this prediction. However, we checked numerically that for large enough values of $Q^{2}$ all curves converge to $\lim _{Q^{2} \rightarrow \infty} Q^{2} F_{\gamma \pi^{0}}\left(Q^{2}\right)=$ $0.2 \mathrm{GeV} \simeq 2 f_{\pi}$. We ascribe the difference of about $8 \%$ between this limit and that of pQCD to our modeling of the transversal component of the pion wave function.

Whilst the hydrogen bound-state model for $m_{u, d}=220 \mathrm{MeV}$ yields a form factor, $Q^{2} F_{\gamma \pi^{0}}\left(Q^{2}\right)$, that comes closest to the BaBar data [1], it does not provide the logarithmic form these new data points seem to imply for $Q^{2}>15 \mathrm{GeV}^{2}$. Moreover, for $Q^{2}<10 \mathrm{GeV}^{2}$, the hydrogen bound-state model reproduces very poorly older CLEO data. If one does not take into account BaBar data points at large four-momentum-squared transfer, the Gaussian wave function with $m_{u, d}=220 \mathrm{MeV}$ provides the best model description of all data including Belle's measurements and coincides with the pQCD limit for large $Q^{2}$. 


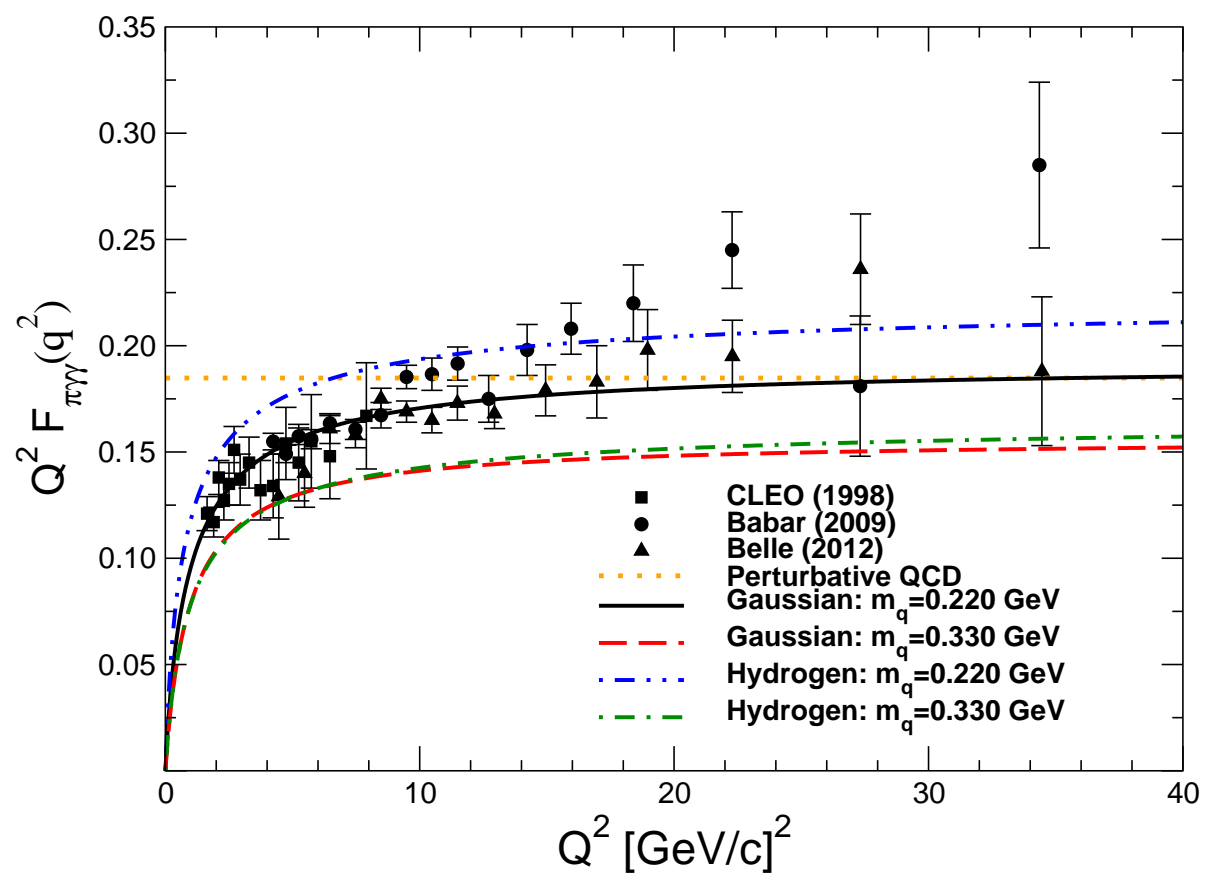

Fig. 3 The momentum-squared weighted transition form factor, $Q^{2} F_{\gamma \pi}\left(Q^{2}\right)$, for two different constituent quark masses $m_{q}=m_{u, d}=M$ and $f_{\pi}=92.4 \mathrm{MeV}$. The dotted horizontal line is the pQCD prediction [5]; see Eqs. (26) and (27) for the two model wave functions and Table 1 for the corresponding length scale parameter, $r_{\mathrm{nr}}$, and charge radii. Data are from Refs. $[1 ; 3 ; 6]$. N.B. For $Q^{2} \rightarrow \infty, Q^{2} F_{\gamma \pi}\left(Q^{2}\right)$ reaches the asymptotic limit $2 f_{\pi}=0.185 \mathrm{GeV}$ for both models and masses.

The elastic form factor for two constituent quark masses and both models is depicted in Figs. 4 and 5. Here, both the hydrogen and Gaussian models with a constituent mass of $220 \mathrm{MeV}$ are in good agreement with data whereas for $m_{u, d}=330 \mathrm{MeV}$ the form factor appears to be too soft. The hydrogen wave function for $m_{u, d}=220 \mathrm{MeV}$ is particularly successful in reproducing the $1 / Q^{2}$ tail of $F_{\pi}\left(Q^{2}\right)$. However, as just noted, the resulting charge radius $\left\langle r_{\pi}^{2}\right\rangle^{1 / 2}=0.795 \mathrm{fm}$ is $20 \%$ larger than the experimental value in this case. On the other hand, this may be a desirable aspect of the model since it is crudely given by a quark-antiquark core with a constant dressed quark mass. It is known, however, that dressing the quark-antiquark scattering kernel with pion loops introduces an attractive force which results in a decrease of the bound state's mass and charge radius (see, e.g., the discussion about pion loop effects in Ref. [45]).

We have tested the present light-front model for various constituent masses from $180 \mathrm{MeV}$ to $330 \mathrm{MeV}$ and find the mass range $220-250 \mathrm{MeV}$ to be the most consistent with a realistic description of the observables discussed herein (for a recent and more detailed discussion, see Ref. [15]). While we acknowledge the mass dependence in our calculations, this is hardly limited to the present light-front model [46]. Indeed, it is a recurrent and unavoidable feature of all constituent quark models which do not account for dynamical chiral symmetry breaking. The use of a constituent quark mass is justified and yields robust results in calculations which involve heavy-quark propagation [47; 48; 49], however this is not true for observables involving light quarks [30; 31].

Nonetheless, this study aims at a consistent treatment of all form factors, the charge radii and decay constant with the same single parameter set, $r_{\mathrm{nr}}$ and $M=m_{u, d}$. We thus employ in the following $m_{u, d}=220 \mathrm{MeV}$ which reproduces well $F_{\pi^{+}}\left(Q^{2}\right),\left\langle r_{\pi}\right\rangle$ and $f_{\pi}$ and yields a satisfying description of $F_{\gamma \pi}\left(Q^{2}\right)$ below $Q^{2} \simeq 15 \mathrm{GeV}^{2}$. 


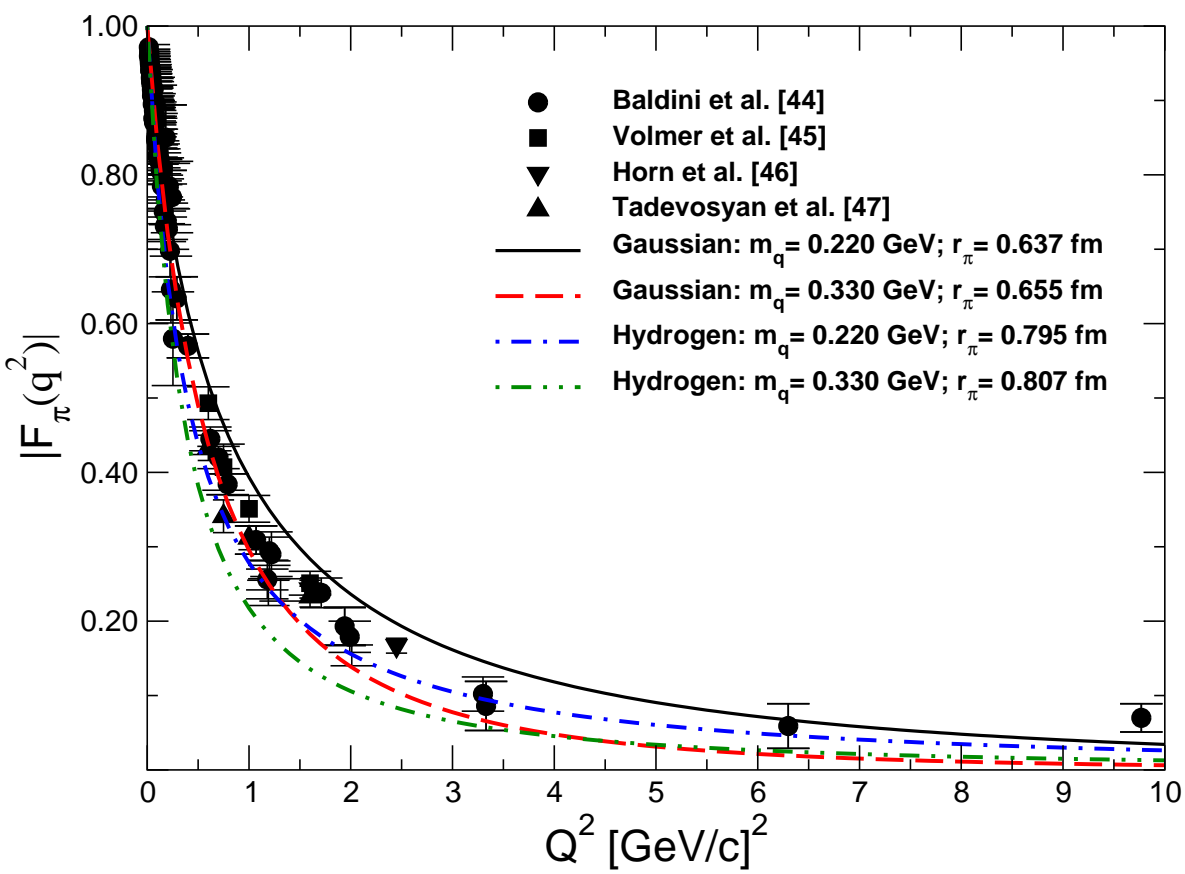

Fig. 4 The space-like elastic form factor, $F_{\pi}\left(Q^{2}\right)$, for two different constituent quark masses $m_{q}=m_{u}=$ $m_{d}=M$ and $f_{\pi}=92.4 \mathrm{MeV}$; see Eqs. (26) and (27) for the two model wave functions and Table 1 for the corresponding length scale parameter, $r_{\mathrm{nr}}$. Data are from Ref. $[50 ; 51 ; 52 ; 53]$.

\section{BaBar data, the pion's length scale and the impact on the charge radius}

Having fixed mass and length scale parameters of the form factors derived in Section 2, we now allow for variation of the latter scale, $r_{\mathrm{nr}}$, while keeping $m_{u, d}=220 \mathrm{MeV}$ fixed. Table 2 illustrates that when $r_{\mathrm{nr}}$ decreases so does the rms charge radius, $\left\langle r_{\pi}^{2}\right\rangle^{1 / 2}$, whereas the weak decay constant, $f_{\pi}$, increases in agreement with relation (18). This is observed independently of the wave function model employed. Bearing this on mind, we modify $r_{\mathrm{nr}}$, in other words the pion width in momentum space, and observe the impact on the form factors discussed in Section 3.

The results for the transition and elastic form factors weighed by $Q^{2}$ are shown for two different charge radii and corresponding decay constants (see Table 2) in Figs. 6 and 7 and for comparison, we plot the form factors for both models. The comparison within Fig. 6 and between Figs. 3 and 6 highlights that the model calculation of $Q^{2} F_{\gamma \pi^{0}}\left(Q^{2}\right)$ coincide increasingly better with BaBar data for smaller charge radii. In particular, the hydrogen type bound-state function for which $f_{\pi}=97 \mathrm{MeV}$, viz. about $5 \%$ larger than in Table 1, yields a transition form factor in reasonable agreement with BaBar data for $Q^{2}>15[\mathrm{GeV} / c]^{2}$. Nonetheless, below $Q^{2} \simeq 15[\mathrm{GeV} / c]^{2}$, this model produces a transition form factor much harder than experimentally evidenced. For the larger decay constant, $f_{\pi}=110 \mathrm{MeV}$, this is even more so the case. At any rate, the wave function model is not appropriate since it neither reproduces the extent CLEO data nor the BaBar data which hints at a logarithmically increasing form factor at large $Q^{2}$.

The best result depicted in Fig. 6 is given by the dashed (blue) line for the Gaussian model and with $f_{\pi}=110 \mathrm{MeV}$ from which we deduce $\left\langle r_{\pi}^{2}\right\rangle^{1 / 2}=0.406[\mathrm{fm}]$, i.e. a pion charge radius $40 \%$ smaller than its experimental value [28]. Decreasing even more $r_{\mathrm{nr}}$ will lead to a harder form factor $F_{\gamma \pi^{0}}\left(Q^{2}\right)$ and improve the agreement with BaBar data, however the corresponding weak decay constant and charge radius turn out to take unrealistic values. Moreover, within the light-cone framework, a wavefunction model congruent with the apparent logarithmic increase of BaBar's $F_{\gamma \pi^{0}}\left(Q^{2}\right)$ data at large $Q^{2}$, e.g. the dashed (blue) line in Fig. 6, is shown in Fig. 7 to fail in reasonably describing the elastic form factor, $F_{\pi}\left(Q^{2}\right)$. The hydrogen-type wave functions yield reasonable rms charge radii and $F_{\pi}\left(Q^{2}\right)$ 


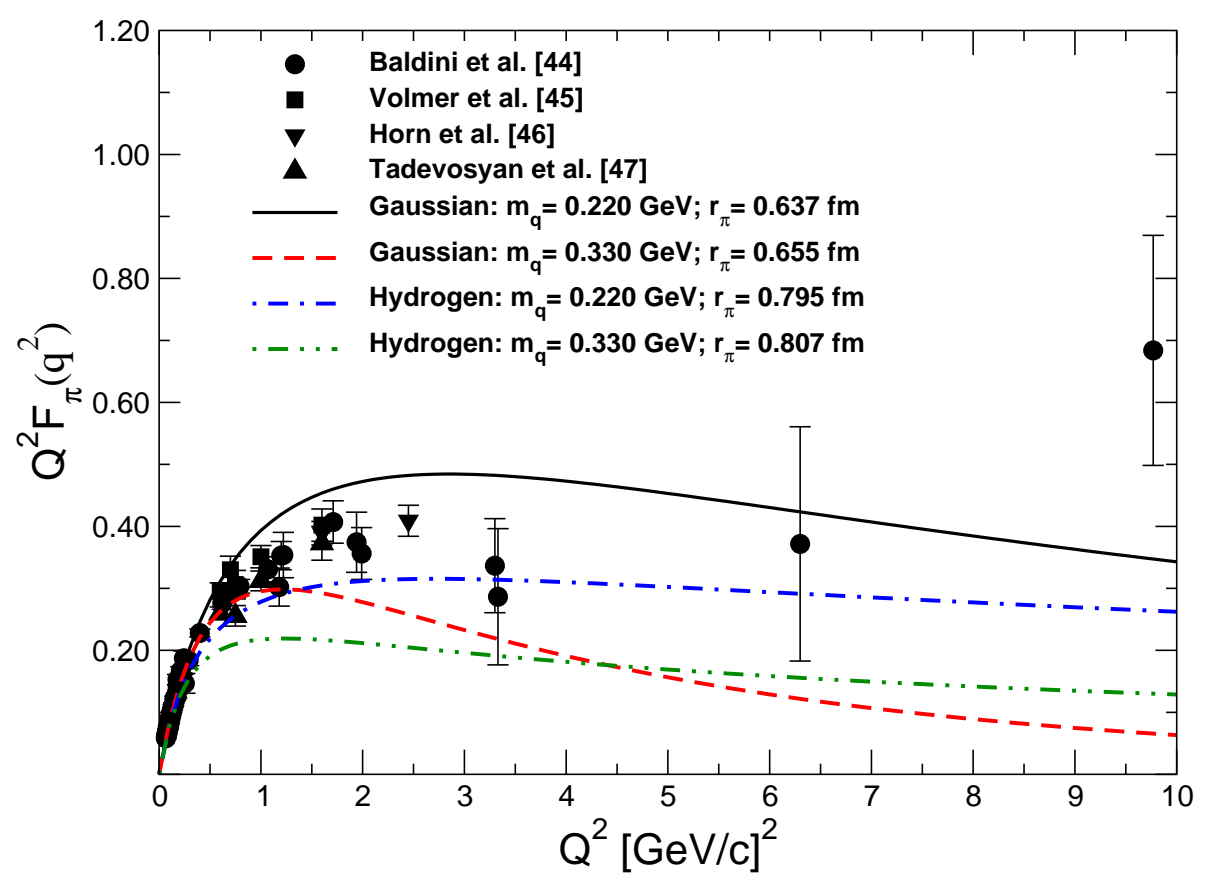

Fig. 5 The momentum-squared weighted elastic form factor, $Q^{2} F_{\pi}\left(Q^{2}\right)$, for two different constituent quark masses $m_{q}=m_{u}=m_{d}=M$ and $f_{\pi}=92.4 \mathrm{MeV}$; see Eqs. (26) and (27) for the two model wave functions and Table 1 for the corresponding length scale parameter, $r_{n r}$. Data are from Ref. $[50 ; 51 ; 52 ; 53]$.

form factors yet, as noted before, the transition form factors are poorly described in most of the $Q^{2}$ domain. Unsurprisingly, here too smaller charge radii are the results of harder form factors which are in disagreement with the current set of data $[50 ; 51 ; 52 ; 53]$ on the elastic pion form factor.

\section{Conclusive Remarks}

A series of recent articles $[16 ; 17 ; 18 ; 19 ; 20 ; 21 ; 22 ; 23 ; 24 ; 31 ; 54 ; 55 ; 56 ; 57 ; 58 ; 59 ; 60]$ have dealt with the discrepancy between BaBar data [1] on $\gamma^{*} \gamma \rightarrow \pi^{0}$ and a long-standing prediction of pQCD [4; 5]. Attempts to reproduce the BaBar data can be generally identified within two broad classes:

- alteration of the asymptotic form of the pion's distribution amplitude or wave function;

- dressing of the $\gamma \bar{q} q$ vertex with phenomenological interactions, such as vector dominance.

As has been discussed extensively in Ref. $[31 ; 55 ; 56 ; 57 ; 58 ; 59]$ the modifications of the pion distribution amplitude, as proposed for instance in Refs. $[16 ; 17 ; 19 ; 20 ; 21 ; 22]$, deviate drastically from its QCD asymptotic form. These altered distributions, $\phi(x)$, are constant or at least non-vanishing for $x=0,1$ and characterize an essentially point-like pion. One comes to a similar conclusion within the framework of a constituent quark model on the light cone or, for the matter, with any other quark model. A faithful reproduction of the BaBar data at large- $Q^{2}$ values leads to a non-vanishing asymptotic pion wave function in momentum space $^{1}$, which we showed to correspond to an excessively small pion charge radius. More generally, if the bound-state wave function does not asymptotically fall off as (at least) $k^{-2}$, the elastic form factor, $F_{\pi}\left(Q^{2}\right)$, becomes harder and may even turn out to take a constant value at large $Q^{2}$. This contradicts the bulk of existing data on the elastic pion form factor. We conclude that it is impossible, within a given consistent approach, to describe $f_{\pi},\left\langle r_{\pi}^{2}\right\rangle^{1 / 2}, F_{\pi}\left(Q^{2}\right)$ and $F_{\gamma \pi}\left(Q^{2}\right)$ equally well. One may alter the pion bound-state function to account for the large- $Q^{2}$ BaBar data; this, however conflicts with other data and QCD-based studies that produce soft pions,

\footnotetext{
${ }^{1}$ at least for practical purposes, as we use Gaussian and hydrogen wave function models which eventually do vanish at large enough $k^{2}$ even for extreme values of $r_{\mathrm{nr}}$.
} 


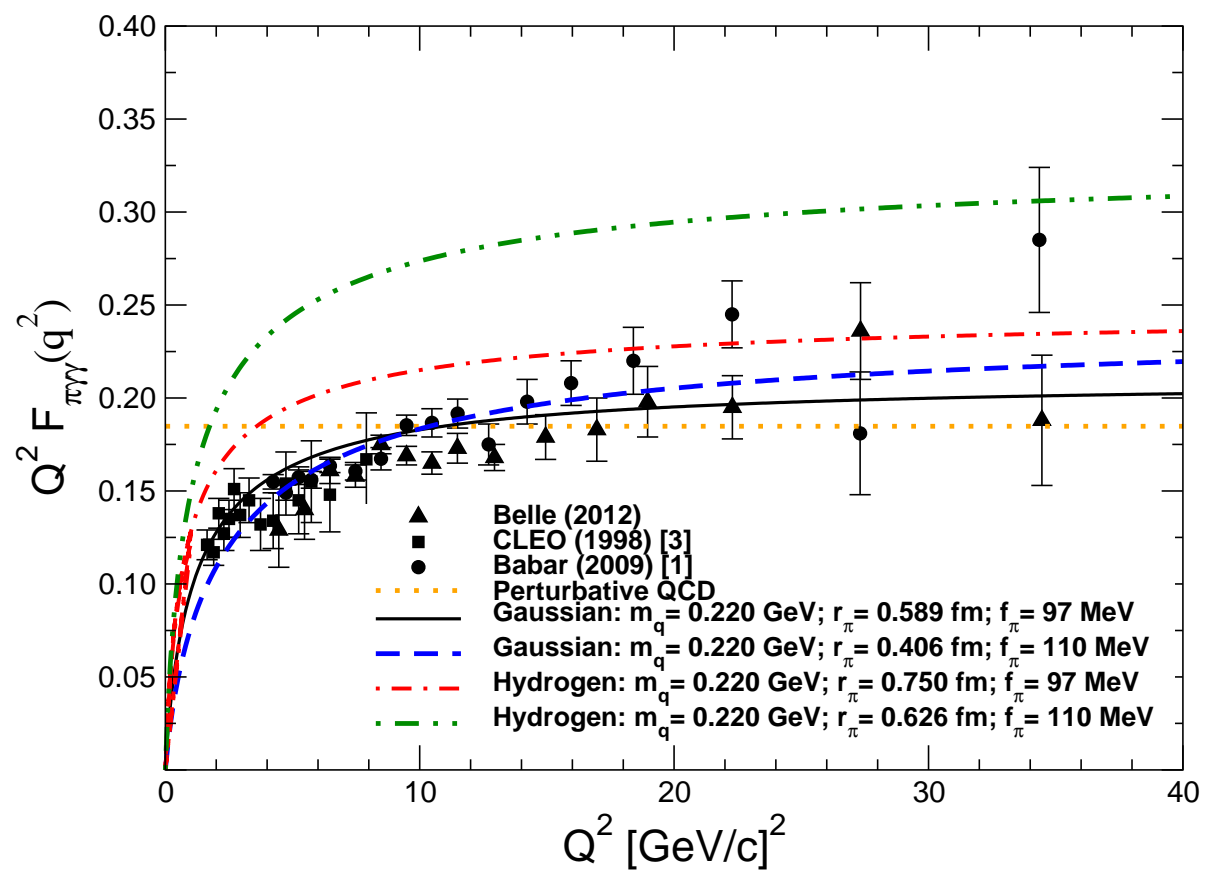

Fig. 6 The momentum-squared weighted transition form factor, $Q^{2} F_{\gamma \pi}\left(Q^{2}\right)$, for $m_{q}=m_{u}=m_{d}=M=$ $220 \mathrm{MeV}$ and two values of $f_{\pi}>92.4 \mathrm{MeV}$. See Table 2 for the corresponding length scale parameter, $r_{\mathrm{nr}}$. Data are from Refs. $[1 ; 3 ; 6]$.

viz. pion distribution amplitudes that vanish as $\sim(1-x)^{2}$ for $x \sim 1$. We also note that our pion transition form factor, the solid line in Fig. 6, is in very good agreement with predictions by Agaev et al. [54], Bakulev et al. [57] and Brodsky et al. [58; 59].

With respect to the photon vertex modification [18; 23; 24], whilst resonances dominate the electromagnetic form factor of the process $\gamma^{*} \rightarrow \pi^{+} \pi^{-}$in the time-like region, this is not necessarily true in the deep space-like domain. It is reasonable to assume the imaginary part of the form factor vanishes at large time-like momentum squared, $s=q^{2} \rightarrow \infty$, and has a unitary cut on the real axis starting at the threshold $s=4 m_{\pi}^{2}$. In this case, the real part is obtained from an unsubtracted dispersion relation,

$$
F_{\pi}(s)=\frac{1}{\pi} \int_{4 m_{\pi}^{2}}^{\infty} d s^{\prime} \frac{\operatorname{Im} F_{\pi}\left(s^{\prime}\right)}{s-s^{\prime}} .
$$

The imaginary part receives contributions from intermediate resonances that dress the quark-photon vertex. Most of these intermediate states, $\gamma^{*} \rightarrow X \rightarrow \pi^{+} \pi^{-}$, lie below $s=10 \mathrm{GeV}^{2}$, the most prominent of which being the $\rho^{0}(770)$. However, the integration in Eq. (28) is over infinite values of $s^{\prime}$ and thus, in averaging out over all possible intermediate states, these resonances are unlikely to have a big impact on the real part of $F_{\pi}(s)$.

Indeed, analyticity and unitarity suggest that higher resonances have little impact on the spacelike elastic form factor even at high momentum transfers, which was shown with a phenomenological light-front constituent quark model that incorporates photon dressing [12; 13]. This model describes the space-like region up to $-10[\mathrm{GeV} / \mathrm{c}]^{2}$, while in time-like region results are reasonable up to $10[\mathrm{GeV} / \mathrm{c}]^{2}$. The approach was developed to calculate the elastic pion form factor in the space- and time-like regions starting from the covariant Mandelstam formula. A vector meson dominance model, built microscopically from the resolvent of a light front mass-squared operator in the spin- 1 channel, was used for the quark-photon vertex dressing. This resolvent is obtained from the vector meson resonance wave functions. The wave functions of both the pion and vector meson resonances are eigenstates of the relativistic constituent quark squared-mass operator [61; 62], which accounts for both confinement through a harmonic oscillator potential and $\pi-\rho$ splitting by means of a Dirac-delta interaction in the 


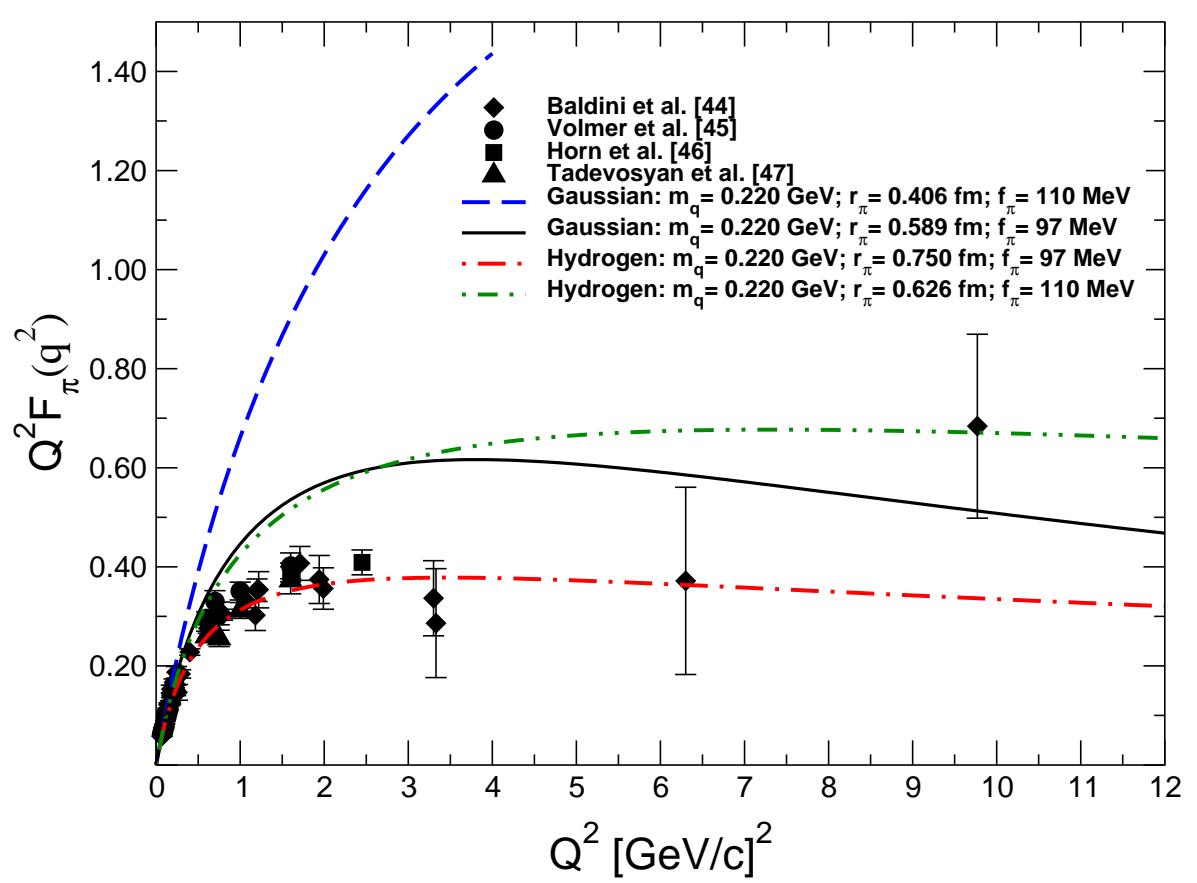

Fig. 7 The momentum-squared weighted elastic form factor, $Q^{2} F_{\pi}\left(Q^{2}\right)$, for $m_{q}=m_{u}=m_{d}=M=220 \mathrm{MeV}$ and two values of $f_{\pi}>92.4 \mathrm{MeV}$. See Table 2 for the corresponding length scale parameter, $r_{\mathrm{nr}}$. Data are from Ref. $[50 ; 51 ; 52 ; 53]$.

pseudoscalar channel. The coupling of the vector resonances to the photon and the decay vertex of the vector meson to the quark-antiquark pair are calculated within the model. The vector decay constants and the overlap between the vector-meson resonances and pion light-front wave functions decrease fast with the radial excitation. This is because the increasing number of nodes in the excited-state wave functions of vector mesons naturally damps both the decay constant as well as the overlap with the pion, strongly suppressing the contribution of the higher resonances to the space-like pion form factor. This behavior can be appreciated in Fig. 9 of Ref. [13] where it is seen that the higher resonances have no influence on the functional behavior of the elastic form factor.

If an analogous model is applied to the $\gamma \gamma^{*} \rightarrow \pi^{0}$ transition form factor, one expects the higher resonances to be suppressed as elucidated by the general discussion based on the dispersion relations in Eq. (28). There is no obvious sign that any amount of dressing the quark-photon vertex with higher resonances can significantly alter the asymptotic $q^{-2}$ decrease of the form factor, whether in the region $Q^{2}=20-40[\mathrm{GeV} / c]^{2}$ or below. Of course, this issue merits further investigation, which we postpone to future scrutiny.

The results of our study suggest the large- $Q^{2}$ BaBar data are inconsistent with other experimental data on form factors, in particular the Belle data, and static properties of the pion. These are, in turn, in agreement with long-standing numerical results of pQCD and soft QCD. Moreover, it is intriguing that the $\gamma^{*} \rightarrow \eta \gamma$ and $\gamma^{*} \rightarrow \eta^{\prime} \gamma$ transition form factors also measured by the BaBar Collaboration at $Q^{2}=112[\mathrm{GeV} / \mathrm{c}]^{2}[63]$ are in full agreement with the CLEO results [3] and theoretical results, see for instance the green band in Fig. 2 of Ref. [57].

Acknowledgements This work was supported by FAPESP grants nos. 2009/00069-5, 2009/53351-0, 2009/512961 and 2010/05772-3 and CNPq grants no. 306395/2009-6 and 305131/2011-7. Constructive and enjoyable discussions with Adnan Bashir, Craig Roberts and Peter Tandy were strongly appreciated. We acknowledge pertinent comments on the manuscript by Nicos Stefanis. 


\section{References}

1. The BABAR Collaboration, B. Aubert et al., Phys. Rev. D80, 052002 (2009), 0905.4778.

2. CELLO Collaboration, H. J. Behrend et al., Z. Phys. C49, 401 (1991).

3. CLEO Collaboration, J. Gronberg et al., Phys. Rev. D57, 33 (1998), hep-ex/9707031.

4. G. R. Farrar and D. R. Jackson, Phys. Rev. Lett. 43, 246 (1979).

5. P. G. Lepage and S. J. Brodsky, Phys. Rev. D22, 2157 (1980).

6. Belle Collaboration, S. Uehara et al., Phys. Rev. D86, 092007 (2012), 1205.3249.

7. T. Frederico and G. A. Miller, Phys. Rev. D45, 4207 (1992).

8. T. Frederico and G. A. Miller, Phys. Rev. D50, 210 (1994).

9. J. P. C. B. de Melo, H. Naus, and T. Frederico, Phys. Rev. C59, 2278 (1999), hep-ph/9710228.

10. J. P. B. C. de Melo and T. Frederico, Phys. Rev. C55, 2043 (1997), nucl-th/9706032.

11. J. P. B. C. de Melo, T. Frederico, H. Naus, and P. Sauer, Nucl. Phys. A660, 219 (1999), hep-ph/9908384.

12. J. P. B. C. de Melo, T. Frederico, E. Pace, and G. Salmè, Phys. Lett. B581, 75 (2004), hep-ph/0311369.

13. J. P. B. C. de Melo, T. Frederico, E. Pace, and G. Salmè, Phys. Rev. D73, 074013 (2006), hep-ph/0508001.

14. B. El-Bennich, J. P. B. C. de Melo, B. Loiseau, J.-P. Dedonder, and T. Frederico, Braz. J. Phys. 38, 465 (2008), 0805.0768.

15. E. O. da Silva, J. P. B. C. de Melo, B. El-Bennich, and V. S. Filho, Phys. Rev. C86, 038202 (2012), 1206.4721 .

16. A. V. Radyushkin, Phys. Rev. D80, 094009 (2009), 0906.0323.

17. A. E. Dorokhov, JETP Lett. 92, 707 (2010).

18. E. R. Arriola and W. Broniowski, Phys. Rev. D81, 094021 (2010), 1004.0837.

19. P. Kroll, Eur. Phys. J. C71, 1623 (2011), 1012.3542.

20. X.-G. Wu and T. Huang, Phys. Rev. D82, 034024 (2010), 1005.3359.

21. T. N. Pham and X. Y. Pham, (2011), 1103.0452.

22. S. S. Agaev, V. M. Braun, N. Offen, and F. A. Porkert, Phys. Rev. D83, 054020 (2011), 1012.4671.

23. P. Lichard, Phys. Rev. D83, 037503 (2011), 1012.5634.

24. M. Gorchtein, P. Guo, and A. P. Szczepaniak, Phys. Rev. C86, 015205 (2011), 1102.5558.

25. D. Melikhov and B. Stech, Phys. Rev. D85, 051901 (2012), 1202.4471.

26. D. McKeen, M. Pospelov, and J. M. Roney, Phys. Rev. D85, 053002 (2012), 1112.2207.

27. C.-C. Lih and C.-Q. Geng, Phys. Rev. C85, 018201 (2012), 1201.2220.

28. Particle Data Group, J. Beringer et al., Phys. Rev. D86, 010001 (2012).

29. A. Bashir et al., Commun. Theor. Phys. 58, 79 (2012), 1201.3366.

30. L. X. Gutiérrez-Guerrero, A. Bashir, I. C. Cloët, and C. D. Roberts, Phys. Rev. C81, 065202 (2010), 1002.1968.

31. H. L. L. Roberts, C. D. Roberts, A. Bashir, L. X. Gutiérrez-Guerrero, and P. C. Tandy, Phys. Rev. C82, 065202 (2010), 1009.0067.

32. H. Roberts, A. Bashir, L. Gutiérrez-Guerrero, C. Roberts, and D. Wilson, Phys. Rev. C83, 065206 (2011), 1102.4376 .

33. R. Dashen and M. Gell-Mann, Phys. Rev. Lett. 17, 340 (1966).

34. S. Fubini, G. Segrè, and J. D. Walecka, Annals Phys. 39, 381 (1966).

35. L. L. Frankfurt and M. I. Strikman, Nucl. Phys. B148, 107 (1979).

36. M. V. Terentev, Sov. J. Nucl. Phys. 24, 106 (1976).

37. P. L. Chung, W. N. Polyzou, F. Coester, and B. D. Keister, Phys. Rev. C37, 2000 (1988).

38. P. L. Chung, F. Coester, and W. N. Polyzou, Phys. Lett. B205, 545 (1988).

39. P. Chung and F. Coester, Phys. Rev. D44, 229 (1991).

40. J. P. B. C. de Melo, T. Frederico, L. Tomio, and A. E. Dorokhov, Nucl. Phys. A623, 456 (1997), hep$\mathrm{ph} / 9708278$.

41. C. Itzykson and J.-B. Zuber, Quantum Field Theory, International Series In Pure and Applied Physics (McGraw-Hill, New York, 1980).

42. L. Ametller, L. Bergström, A. Bramon, and E. Massó, Nucl.Phys. B228, 301 (1983).

43. R. Tarrach, Z. Phys. C2, 221 (1979).

44. M. G. Fuda, Annals of Physics 197, 265 (1990).

45. L. Chang, I. C. Cloët, B. El-Bennich, T. Klähn, and C. D. Roberts, Chin. Phys. C33, 1189 (2009), 0906.4304 .

46. D. Melikhov, Eur. Phys. J. direct C4, 2 (2002), hep-ph/0110087.

47. B. El-Bennich, M. A. Ivanov, and C. D. Roberts, Phys. Rev. C83, 025205 (2011), 1012.5034.

48. B. El-Bennich, G. Krein, L. Chang, C. D. Roberts, and D. J. Wilson, Phys. Rev. D85, 031502 (2012), 1111.3647.

49. B. El-Bennich, C. D. Roberts, and M. A. Ivanov, (2012), 1202.0454.

50. R. Baldini et al., Nucl. Phys. A666, 38 (2000).

51. The Jefferson Lab F(pi) Collaboration, J. Volmer et al., Phys. Rev. Lett. 86, 1713 (2001), nucl-ex/0010009.

52. Jefferson Lab F(pi)-2 Collaboration, T. Horn et al., Phys. Rev. Lett. 97, 192001 (2006), nucl-ex/0607005.

53. Jefferson Lab F(pi) Collaboration, V. Tadevosyan et al., Phys. Rev. C75, 055205 (2007), nucl-ex/0607007.

54. S. Agaev, V. Braun, N. Offen, and F. Porkert, Phys. Rev. D86, 077504 (2012), 1206.3968.

55. N. Stefanis, Few Body Syst. 52, 415 (2011), 1109.2718.

56. A. Bakulev, S. Mikhailov, A. Pimikov, and N. Stefanis, Phys. Rev. D84, 034014 (2011), 1105.2753.

57. A. Bakulev, S. Mikhailov, A. Pimikov, and N. Stefanis, Phys. Rev. D86, 031501 (2012), 1205.3770.

58. S. J. Brodsky, F.-G. Cao, and G. F. de Teramond, Phys. Rev. D84, 033001 (2011), 1104.3364. 
59. S. J. Brodsky, F.-G. Cao, and G. F. de Teramond, Phys. Rev. D84, 075012 (2011), 1105.3999.

60. I. Balakireva, W. Lucha, and D. Melikhov, Phys. Rev. D85, 036006 (2011), 1110.6904.

61. T. Frederico, H.-C. Pauli, and S.-G. Zhou, Phys. Rev. D66, 054007 (2002), hep-ph/0207207.

62. T. Frederico, H.-C. Pauli, and S.-G. Zhou, Phys. Rev. D66, 116011 (2002), hep-ph/0210234.

63. BABAR Collaboration, B. Aubert et al., Phys. Rev. D74, 012002 (2006), hep-ex/0605018. 